\title{
Crystallization of Partially Amorphized Ni-Mo Alloy
}

\author{
I. Estrada-Guel ${ }^{1}$, F. Espinosa-Magaña ${ }^{1}$, L. Bejar Gómez ${ }^{2}$, J.I Barajas-Villaruel ${ }^{3}$ and R. Martínez- \\ Sánchez ${ }^{1 *}$. \\ ${ }^{1}$ Centro de Investigación en Materiales Avanzados (CIMAV), Miguel de Cervantes No.120, C.P. 31110, Chihuahua, \\ Chih., México. \\ ${ }^{2}$ Universidad Michoacana de San Nicolás de Hidalgo, Edif.-W; C.U, C.P 58000, Morelia, Mich. México. \\ ${ }^{3}$ Universidad Autónoma de San Luis Potosí, Alvaro Obregón No. 64, C.P. 78000, San Luis Potosí, S.L.P., México.
}

The Ni-Mo system has been widely investigated by the mechanical alloying (MA) research community. The phase transformation in amorphous Ni-Mo alloys obtained by MA has also been reported. In many cases it has been established that amorphization of the material is the final state of the powder subjected to the milling action. Complete or partial amorphization in this binary system mainly depends on the concentration of the elements. This work is focused to the characterisation of amorphous structures obtained by mechanical alloying in a Mo-47 at. \% Ni alloy, and their phase transformations under heat treatment This composition corresponds to the equiatomic NiMo intermetallic. The characterisation was carried-out by XRD, SEM-EDS, DTA and TEM.

Collected XRD patterns (in Figure 1) shows partial amorphized materials after $36 \mathrm{~h}$ milling. Apparently, this is a preferential $\mathrm{Ni}$ amorphization. In all XRD patterns it is clear that the typical diffraction peaks $(2 \theta \cong 40$ degrees) corresponding to Mo suffered shortening and broadening but not shifting. This latter evidence indicates that Mo it is not dissolving Ni nor Fe (from milling media wear).

Figure 2 shows a representative view TEM picture and their SADP from particle in the asmilled condition.

After heating the as-milled powder, XRD analysis revealed microstructural transformation and formation of secondary phases. Fig. 3 shows the presence of $\mathrm{MoO}_{2}$, "Y" phase, NiO, and Mo.

In this work, an oxicarbide $\left(\mathrm{Ni}_{6} \mathrm{Mo}_{6}(\mathrm{C}, \mathrm{O})_{1.06}\right)$ has been identified in samples in the asheated condition and has been found as the crystallised phase in major amount in the final product. The XRD pattern of this oxicarbide is very similar to that reported before for a new phase called "Y". The simulated XRD pattern of oxicarbide $\left(\mathrm{Ni}_{6} \mathrm{Mo}_{6}(\mathrm{C}, \mathrm{O})_{1.06}\right)$ is in good agreement with that experimentally found in this work. Although the " $\mathrm{Y}$ " phase formation has been attributed to the presence of $\mathrm{C}, \mathrm{O}$ or $\mathrm{N}$ contaminants, its identification and characteristics remains unclear.

From those XRD patterns it seems that the precipitated phases formed after heating are essentially the same independently of the heating time. This indicates that there is a high thermodynamic stability of the crystallised phases at such heating conditions. Figure 4 shows a representative view from a crystalline particle after heat treatment.

\section{Acknowledgement}

This work was supported by CONACYT (J32620-U). 


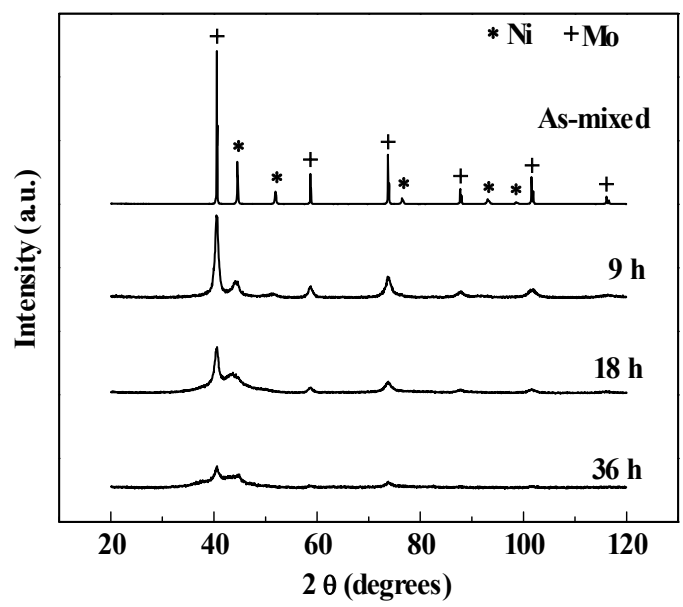

Figure 1.- XRD spectra for Ni-Mo mixtures milled in SPEX mill for different times in a SS container under argon atmosphere.

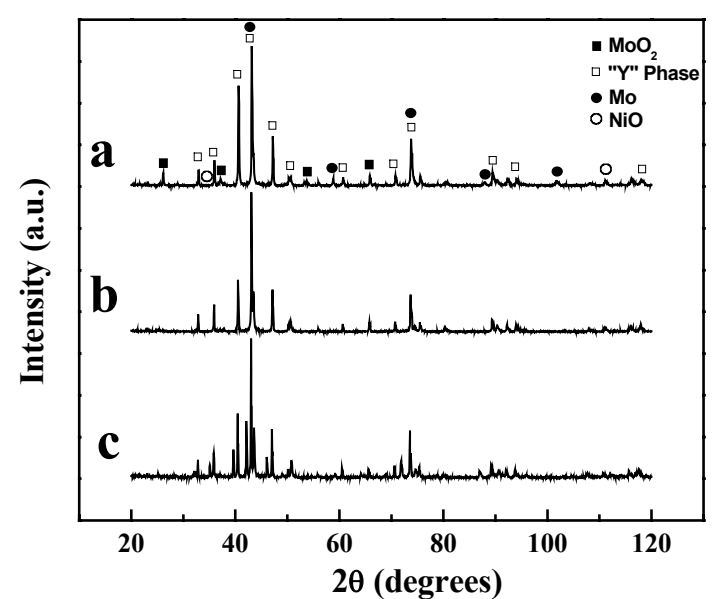

Figure 3.- XRD patterns from samples milled during different times, a) $9 \mathrm{~h}$, b) 18 $\mathrm{h}$ and c) $36 \mathrm{~h}$.

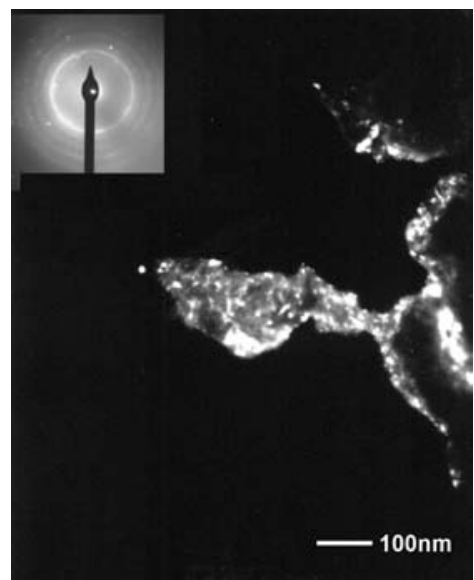

Figure 2.- TEM picture and their SADP from particle in the as-milled condition, the inset square shows an amorphous halo.

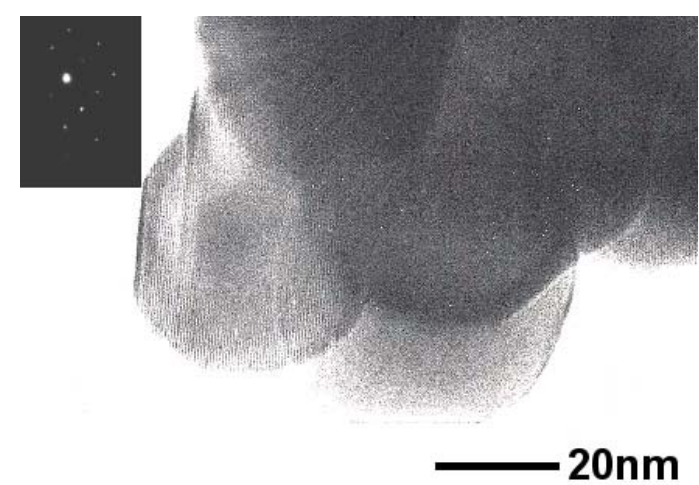

Figure 4.- TEM picture and their SADP in NiMo particle after heat treatment. 